\title{
The Development of a New, Rapid, Amperometric Immunosensor for the Detection of Low Concentrations of Bacteria Part II: Optimization of the System for Escherichia coli
}

\author{
Eric Carnes and Ebtisam Wilkins \\ Department of Chemical and Nuclear Engineering, University of New Mexico \\ Albuquerque, New Mexico 87131
}

\begin{abstract}
The optimization of a flow-through immunoassay system was conducted to achieve rapid detection of low concentrations of $E$. coli bacteria. The system includes a new immunosensor that uses newly developed, disposable immunofiltration membranes to yield a system that is sensitive as well as simple. Comparison of the performance of this system with conventional immunoassays demonstrated a significantly shorter time for the assay. Optimization of the parameters of the system, including specific flow rates and concentrations, was conducted, resulting in an overall assay time of $17 \mathrm{~min}$

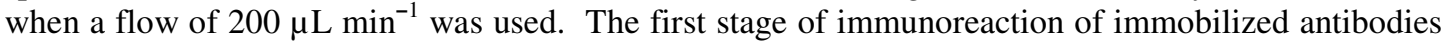
with the analyte was found to saturate in $2 \mathrm{~min}$. The second stage of immunoreaction with the enzyme-labeled conjugate exhibited a more complex behavior but became saturated in 6 min. The amperometric detection using the substrate gave a steady output signal after $3 \mathrm{~min}$. All stages are separated by 2 min of washing by a rinsing buffer solution and the whole analysis is preceded by a 2minute pre-washing stage in order to eliminate impurities which can lead to non-specific binding of immunoagents and substantial background noise. The linear working range for the immunoassay was found to be $50-1000$ cells $\mathrm{mL}^{-1}$. The disposable immunofiltration membranes were found to retain $75 \%$ of their activity over a period of 7 days when refrigerated at $4^{\circ} \mathrm{C}$. The short assay time, in combination with the ability to detect small concentrations of bacteria, permits the application of the developed system to near-real time detection of a wide range of analytes in many different applications, such as medical diagnostics, environmental applications and food quality control.
\end{abstract}

Key words: Immunoassay, Sensitive, Near Real-time, Biosensor, Carbon Paper, Immunofiltration

\section{INTRODUCTION}

Enterobacteriaceae form a large, heterogeneous family of medically and scientifically important, gramnegative bacteria. Escherichia coli, the most prevalent infecting organism in this family, is one of the prototypic bacteria studied [1]. Members of the family are widely distributed in the soil and on plants and are normal colonizers of the human intestinal tract. However, many genera in the family are major human intestinal pathogens, like Shigella and Salmonella. As a result, Enterobacteriaceae are commonly referred to as "enteric bacteria" because of the association with intestinal colonization, even though many other families of bacteria are commonly found in the human gastrointestinal system.

Although not usually found as a component of the normal flora outside of the gastrointestinal tract, Enterobacteriaceae are an important cause of infections elsewhere in the body. In hospitalized or immunocomprimised individuals, colonization with gram-negative bacteria does occur [2]. These infections frequently lead to pneumonia, septicemia, meningitis, or abscess formation. These bacteria account for approximately $80 \%$ of significant isolates of gram- negative bacteria in the clinical laboratory [3]. It has been estimated that Enterobacteriaceae account for about one-third of the septicemia isolates, two-thirds of the bacterial gastroenteritis isolates and three-fourths of the urinary tract isolates [4].

$E$. coli is generally acknowledged as the best-studied free-living organism [5]. These bacteria can be either motile or non-motile and most ferment lactose. In order to detect the bacteria, a sample must be cultured using sorbitol-MacConkey agar, which usually takes a couple of days to obtain a usable culture before tests can be performed. Laboratory tests frequently used to identify $E$. coli include a positive reaction with methyl red, a Voges-Proskauer test that yields negative results and the lack of activity of phenylalanine deaminase as well as urease [6]. This bacterium is the most frequent cause of some of the most common bacterial infections, including urinary tract infections, bacteremia, traveler's diarrhea and even neonatal meningitis. It can also cause a variety of other clinical infections, including pneumonia. Although E. coli normally colonizes the large intestine, virulent serogroups are not typically found in fecal samples of healthy human adults. Rather, infections tend to be found in the urinary tract, meninges and blood stream [7]. 
As early as the 1920's, E. coli was known to cause human infantile diarrhea [8]. It has recently been recognized as the major cause of bacteria gastroenteritis in connection with traveling abroad [9]. At least five varieties of $E$. coli can cause enteric infections, which ease hemisphere tending to have its own type [10]. Traveler's diarrhea exhibits symptoms of abdominal cramps and frequent, explosive bowel movements as a result of large amounts of fluid being released from the gastrointestinal tract. The disease usually arises in a healthy individual from an industrialized nation visits a non-developed country, which are often characterized by poor hygiene. The enterohemorrhagic E. coli usually belong to the serotype 0157:H7 and were first associated with a multi-state outbreak in 1982 [11]. Antibiotics have been effectively used to combat traveler's diarrhea, but because of the increase in drugresistant bacteria, it is probably best for the traveler to use extreme care in their consumption of food and water that may be contaminated. Once a person is infected, it is extremely important to replace the fluids and electrolytes that are lost through diarrhea.

Even though the most common site for $E$. coli colonization is in the gastrointestinal system, the most common site for infection is the urinary tract. This is because the urinary tract is normally sterile and any intrusion of bacteria can reproduce without competition. E. coli is the most common cause of urinary tract infections that can result in a wide range of illnesses, including urethritis to symptomatic cystitis [12]. Infection is prevalent in women due to a shortened urethra and close proximity to the periurethral region, which contains a high number of coliform bacteria. The most important host factor appears to be the presence of a foreign body, like a catheter, which obstructs normal urinary flow and gives opportunistic organisms, like $E$. coli, a chance to thrive. Antibiotics are usually given to shorten the infection time, but they are not always effective and often the infection simply runs its natural course [13].

Intra-abdominal infection can result in two major clinical manifestations. Early or diffuse infection results in localized or generalized peritonitis. Late and localized infection produces an abscess. Presently, peritonitis and related infections are the second leading cause of death in dialysis patients. E. coli is one of the major types of bacteria responsible for infection leading to bacterial peritonitis. Primary peritonitis occurs most usually in adolescent girls and is characterized by a diffuse bacterial infection without loss of integrity of the gastrointestinal tract. Secondary peritonitis, often referred to as acute peritonitis, results in perforations of the gastrointestinal tract, along with anastomotic dehiscence and possible infected pancreatic necrosis. Treatment of this disease involves extensive antibiotic treatment as well as surgery to remove the source of the infection and any other severely inflamed surrounding areas [14].
Unlike gastrointestinal infections in which $E$. coli express themselves as pathogens, the tendency of the bacteria to be opportunistic results in respiratory tract infections. This means that normal, healthy people are susceptible to gastrointestinal and urinary tract infections, while infection of the respiratory tract usually occurs in a person that is already weakened with another illness. It has been shown that severely ill people have an altered physiology that allows colonization of the bacteria [15]. Patients such as diabetics, alcoholics and those with chronic pulmonary disease are much more susceptible to E. coli-related pneumonia than generally healthy people. Treatment is similar to other pneumonias, including use of antibiotics and draining of any extracellular fluids.

In their first months of life, infants are especially susceptible to bacterial meningitis. E. coli is one of the prime culprits of infection in the majority of cases [16]. Infection is thought to be brought about either through pregnancy or by way of the gastrointestinal tract and into the blood stream, where colonization occurs. Treatment usually requires extensive antibiotic therapy. Invasive bacteremia from $E$. coli is fairly uncommon, even though colonization of the bacteria in the gastrointestinal lining is frequent. This is because most strains of $E$. coli are not invasive and are filtered out by the liver and spleen. However, bacteremia can occur in conjunction with a urinary tract infection and can lead to life threatening responses of shock and decomplementation. E. coli was one of the leading causes of bacteremia in the 1970's, but has steadily declined and now accounts for less than one-tenth of reported cases [17].

In this study, a new immunosensor has been developed that seeks to quantify small concentrations of $E$. coli in near-real time. Total E. coli was used as a model to show the system's feasibility to detect E. coli O157: H7. The optimized immunoassay procedure can measure concentrations as low as 50 cells $\mathrm{mL}^{-1}$ in 17 $\min$.

\section{MATERIALS AND METHODS}

Immunoassay System Design Concepts: The heterogeneous immunoassay system design concept is based on conducting enzyme-linked assays by utilizing flow-through immunofiltration membranes coupled with electrochemical-based transduction as a means of overcoming the limitations associated with conventional techniques. A sandwich scheme is utilized using horseradish peroxidase labels to enable electrochemical detection. The immunoassay system consists of a cassette pump with variable-rate flow control, the immunosensor, a data acquisition system and bottles for reagent and waste storage.

Immunofiltration Membranes: A design based on the use of [18] carbon paper as the solid support for the 
immunofiltration membrane has been described in detail in Part I. The carbon paper has many benefits, including pores that can filter out the bacteria and intrinsic pores that make the carbon an ideal immunosorbent, as well as also being conductive and having a high surface area to volume ratio. The use of an immunofiltration membrane facilitates the concentration of the analyte from sample volumes of a dilute concentration by localizing when the immunoreactions take place. A membrane constructed of a porous substance would increase the ratio of reactive surface area to the volume of the liquid media in which the immunoagents are present, resulting in higher sensitivity. Use of an immunosorbent substance allows a large amount of antibodies to be immobilized on the surface, increasing the reactivity of the surface and thereby increasing the sensitivity of the assay.

Immunosensor: The immunosensor design is based on a combination of a flow-through technique with an immunofiltration membrane that also serves as an immunoelectrode and has been discussed in Part I. The disposable immunofiltration membrane made of Toray carbon paper has already been described in detail. The sensor casing is constructed of silastic and Teflon plastics and is designed to allow for a range of flow rates, while maintaining the greatest possible area for interaction of the various immunoagents at the immunofiltration membrane surface. The use of Teflon plastic ensures that none of the immunoagents absorb into the sensor casing, which could cause interference or an unreliable response.

The immunosensor also utilizes a three-electrode system to provide more stable and reliable measurements. In a three-electrode system, besides the working and reference electrodes, a counter electrode is introduced. This gives a true reference electrode for controlling the potential and a counter electrode for current injection. This results in a system which gives much more accurate results when operating at high sensitivity levels.

Carbon paper (TGP-H-1.0t) was a courtesy of Toray Composites, Inc. (Tacoma, WA). Carbon rods, 1/16 in. diameter, used as electrodes and current collectors were a courtesy of DFI Pultruded Composites Inc. (Erlanger, KY). Silver discs, $1 \mathrm{~mm}$ thick, used for reference electrodes was from Thunderbird Supply (Albuquerque, NM). Woodward's reagent K (N-ethyl-5-phenylisoxazolium-3'-sulfonate), trypsin inhibitor, sodium chloride, sodium iodide, hydrogen peroxide and Tween 20 were obtained from Sigma Chemical Company (St.Louis, MO). All chemicals used were of analytical grade. Affinity purified antibody to total E.coli and peroxidase-labeled affinity purified antibody to total E.coli (conjugate) were obtained from Virostat (Portland, MA). Distilled water was used for the preparation of all aqueous solutions.
Preparation of Immunofiltration Membranes: Preparation of the immunofiltration membranes was adapted from a procedure designed for graphite particles [19]. To first activate the carbon paper, a 20 $\mathrm{mg} \mathrm{mL} \mathrm{mL}^{-1}$ solution of Woodward's Reagent $\mathrm{K}$ is prepared in water. Next, discs of Toray carbon paper (TGP-H-1.0t) with 7/8-inch diameter are placed in separate glass bottles and immersed in $1 \mathrm{~mL}$ of the above solution. The discs are then incubated while being shaken for $2 \mathrm{~h}$ at room temperature. Following the incubation, the discs are washed 3 times, for $3 \mathrm{~min}$ each time, with $1 \mathrm{~mL}$ of PBS ( $\mathrm{pH} 7.8)$. Then the discs are transferred to a polystyrene plate and allowed to dry. Next, $500 \mu \mathrm{L}$ of a predefined concentration of antibody solution is added to each well and allowed to incubate for $8 \mathrm{~h}$ at $4^{\circ} \mathrm{C}$. Finally, each disc is incubated with shaking for $2 \mathrm{~h}$ in $1 \mathrm{~mL}$ of a solution of $5 \mathrm{mg} \mathrm{mL}^{-1}$ of trypsin inhibitor (as a blocking agent) in PBS ( $\mathrm{pH}$ 7.8). The discs are again washed as described above and stored in $1 \mathrm{~mL}$ of $\mathrm{PBS}(\mathrm{pH} 7.8)$ at $4^{\circ} \mathrm{C}$ until use.

\section{RESULTS AND DISCUSSION}

The immunoassay system was optimized for the target analyte, $E$. coli, over a working range of 50-1000 cells $\mathrm{mL}^{-1}$, which covers many government-mandated limits, by investigating parameters such as flow rate and reagent concentration. Optical density tests were used to determine an optimum thickness of carbon paper to be used for the immunofiltration membranes. It was desired to have membranes constructed of paper that would filter out at least $50 \%$ of an E. coli sample passed through them. It was found using $1 \mathrm{~mm}$ thick carbon paper, which filters out $66 \%$ of the sample, would best meet this criteria.

The working potential for the amperometric measurements was determined from a cyclic voltammetry experiment using iodine, the product of the enzymatic reaction of the substrate and the carbon paper to be used for the immunofiltration membranes. From the results of a cyclic voltammograph (Fig. 1), it was found that using a working potential of 0.150 Volts would provide the largest difference in output response for different concentrations of iodine. The concentration of sodium iodide/hydrogen peroxide substrate was found to be optimum at $0.4 \mathrm{mM}$. This provided for the largest signal output while maintaining a concentration low enough that it would not be able to effectively auto-oxidize itself or damage the other immunoagents present in the system.

The flow rate to be used in the system was then optimized. A single flow rate would provide simple operation of the immunoassay system and allow for the system to be easily automated. A substrate flow rate of $200 \mu \mathrm{L} \mathrm{min}{ }^{-1}$ was found to give the largest current output relative to the total volume used (Fig. 2). This flow rate was chosen for the rest of the stages in order 


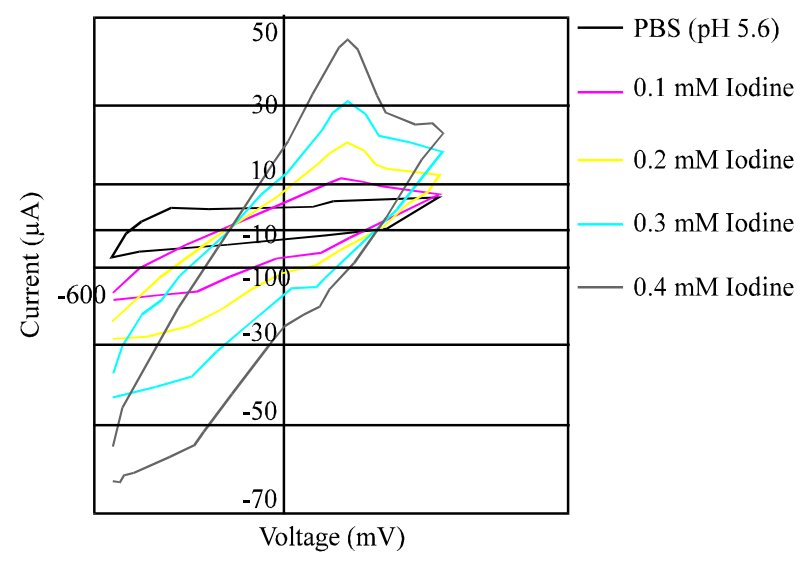

Fig. 1: CV of Iodine in PBS (pH 5.6) with Carbon Electrodes and $\mathrm{Ag} / \mathrm{AgCl}$ Reference

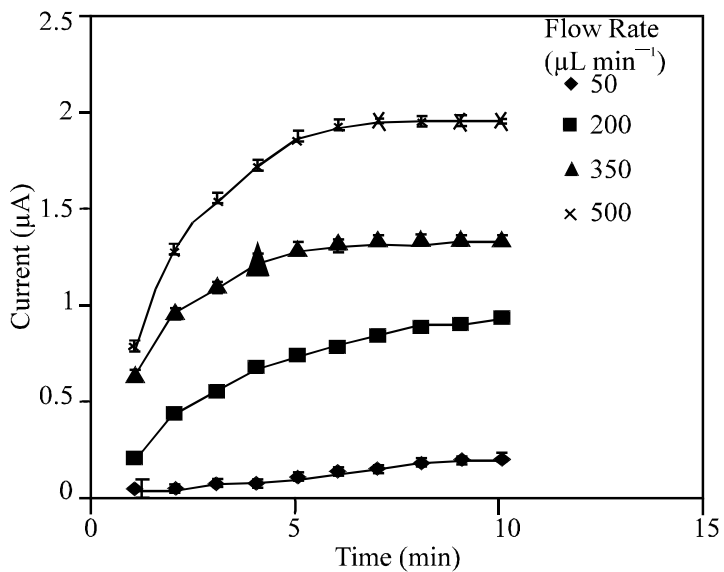

Fig. 2: Effect of Different Flow Rates of Iodine $(0.4 \mathrm{mM})$ on Amperometric Response of Immunoassay System

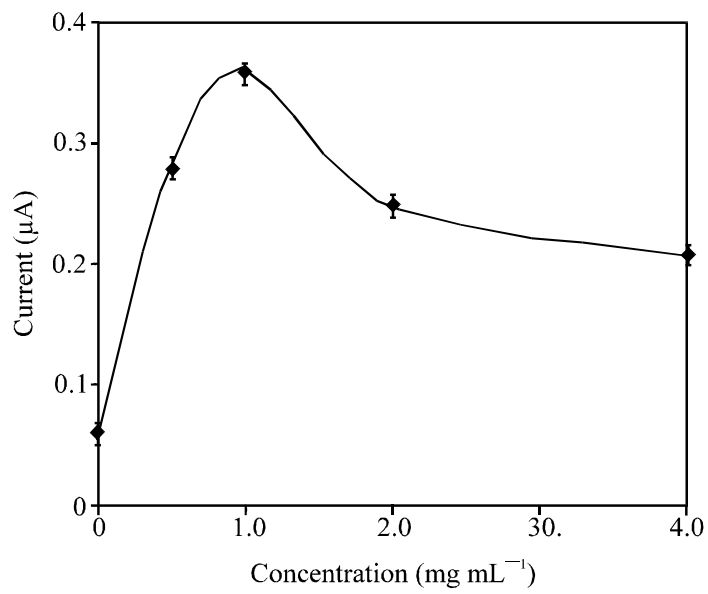

Fig. 3: Effect of Immobilized Antibody Concentration on Output Current Using $0.4 \mathrm{mM}$ Substrate

to simplify the procedure and aid in future automation of the system.
The capacity of the carbon paper for immobilization of antibodies was studied by immobilizing HRP-labeled antibodies and then measuring the signal when substrate was passed over them (Fig. 3). A concentration of $1 \mathrm{mg} \mathrm{mL}^{-1}$ of antibodies gave the highest output. Since this concentration was a 1:4 dilution from the stock solution, it was also determined to be optimum since the expensive antibodies were more efficiently used. The concentration of the immunoconjugate had been previously studied [20] and an optimum of $0.08 \mathrm{mg} \mathrm{mL} \mathrm{m}^{-1}$ was used for this immunoassay system.

The duration of flow of the analyte was then optimized for the upper bound of the desired working range, 1000 cells $\mathrm{mL}^{-1}$. It was found that saturation of the system occurred at this maximum desired concentration of analyte after 2 min (Fig. 4). An additional incubation time, with no flow, to ensure complete equilibrium of the reaction was also investigated. It was found to produce only marginally higher signals, possibly because of the large active surface area available for the reaction. It was therefore decided that an additional incubation time with no flow would not be necessary for the first stage in the sandwich scheme.

The duration of immunoconjugate flow was then investigated to optimize the system response at 1000 cells $\mathrm{mL}^{-1}$ (Fig. 5). The response was found to increase until the duration of immunoconjugate flow reached 6 min, after which the immunofiltration membrane became saturated and no further increase in response was produced. It was also thought that an incubation time for the immunoconjugate would allow the immunoreaction to proceed further, yielding increased sensitivity. However, it was found that an incubation time had no affect on the response of the system, most likely due to the ability of the reaction to proceed efficiently due to the large size of the bacteria that have binded with the immobilized antibodies relative to the immunoconjugate.

The problem of contaminants in the system which could lead to non-specific binding of immunoagents was addressed through the use of washing stages [21]. Washing stages, consisting of passing a rinsing buffer solution for two min, were used in between all stages to rinse away non-bound immunoagents. A pre-washing stage of two min was also introduced to eliminate any background signal from residual immunoagents of prior assays.

After investigating the various parameters that affect the immunoassay, the following optimized procedure was determined:

* Pre-washing stage: flow of a rinsing buffer solution, PBST ( $\mathrm{pH} 7.8$ ), for a duration of $2 \mathrm{~min}$

* First stage of immunoreaction: flow analyte in PBS ( $\mathrm{pH}$ 7.8) for a duration of $2 \mathrm{~min}$ 


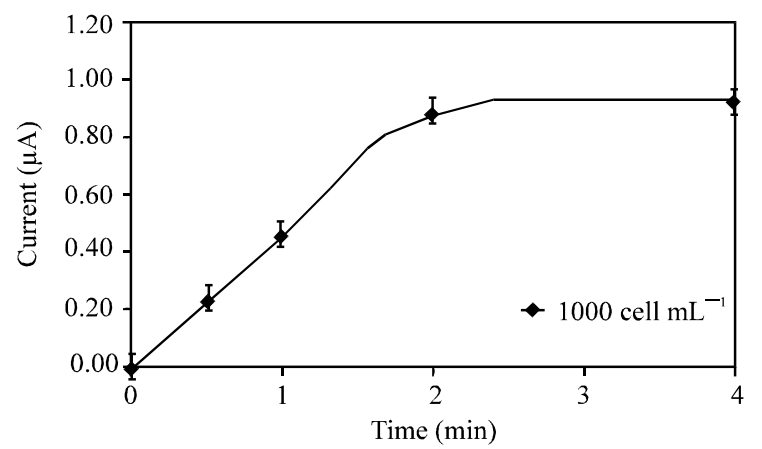

Fig. 4: System Response for Various Analyte Flow Times $\left(1000\right.$ cells $\left.\mathrm{mL}^{-1}\right)$ with $2 \mathrm{~min}$ of Immunoconjugate Flow Using $0.08 \mathrm{mg} \mathrm{mL}^{-1}$ Immunoconjugate and $0.4 \mathrm{mM}$ Substrate

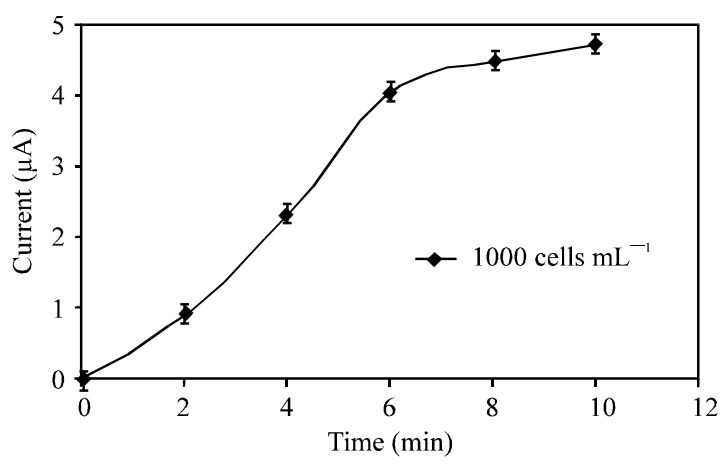

Fig. 5: System Response for Various Immunoconjugate $\left(0.08 \mathrm{mg} \mathrm{mL}^{-1}\right)$ Flow Times with 2 min of Analyte Flow (1000 cells $\mathrm{mL}^{-1}$ ) Using $0.4 \mathrm{mM}$ Substrate

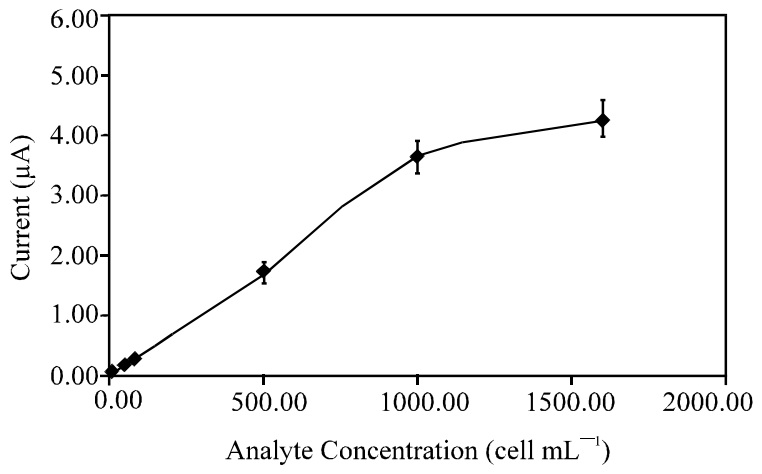

Fig. 6: Calibration Curve for E. coli Using Optimized Immunoassay Procedure Showing Linear Dependence on Concentration over Working Range (50-1000 cells $\mathrm{mL}^{-1}$ )

* First washing stage: flow of PBST (pH 7.8) alone for a duration of $2 \mathrm{~min}$

* Second stage of immunoreaction: flow of immunoconjugate $\left(0.08 \mathrm{mg} \mathrm{mL}^{-1}\right)$ in PBS ( $\left.\mathrm{pH} 7.8\right)$ for a duration of $6 \mathrm{~min}$

* Second washing stage: flow of PBST (pH 5.6) for a duration of $2 \mathrm{~min}$

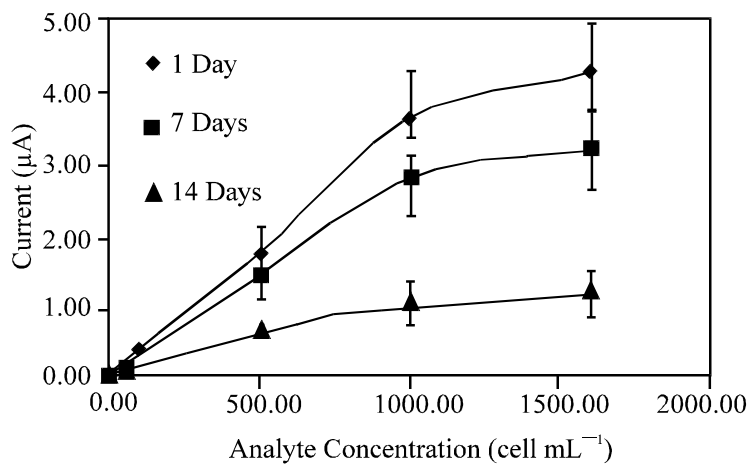

Fig. 7: Effect of Incubation Time on Activity of Immunofiltration Membranes Stored at $4^{\circ} \mathrm{C}$ Using Optimized Conditions

* Amperometric measurement stage: flow of substrate $(0.4 \mathrm{mM})$ in PBS ( $\mathrm{pH} 5.6$ ) for $3 \mathrm{~min}$. Amperometric output of iodine formed as a result of the enzymatic oxidation of iodide is recorded under a $150 \mathrm{mV}$ bias.

The overall assay time was found to be $17 \mathrm{~min}$. This optimized assay could then detect the target analyte over a range of $50-1000$ cells $\mathrm{mL}^{-1}$ in a very linear fashion (Fig. 6). Higher concentrations of analyte could be analyzed, however, the results were not linear. This indicates there is a saturation point in the system that is slightly higher than the maximum value of the desired working range.

The longevity of the immunofiltration membranes was investigated by attempting to repeat the calibration curve with membranes that had been stored at $4^{\circ} \mathrm{C}$ for different durations of time (Fig. 7). It was found that the membranes maintain $75 \%$ of their activity for one week, after which the activity rapidly declines. It was determined, therefore, that this study met the desired objectives and produced a rapid and sensitive immunoassay system for the detection of pathogens consisting of a new immunosensor utilizing disposable immunofiltration membranes that also serve as immunoelectrodes. The system can also be made portable and automated, which opens the door for future development. Another beneficial feature would also be to have a membrane which could be reusable.

This system also compares well to other available immunosensor technologies. Many novel sensors are constantly being developed, especially for the detection of different pathogens and contaminants. The sensor described in this work is designed to assay for E. coli, a common strain of bacteria that can be infectious and pathogenic. Other recent sensors have been developed that assay for similar bacteria, like Salmonella or Listeria [22].

One electrochemical sensor, that is designed to aid in biosecurity, uses lateral flow and a sandwich scheme to capture E. coli or Salmonella and measures the change 
American J. Applied Sci., 2 (3): 607-613, 2005

Table 1: Comparison of Recently Developed Biosensors for the Detection of Bacteria

\begin{tabular}{|c|c|c|c|c|c|}
\hline System description & Application & Range of detection & Analysis time & \multicolumn{2}{|c|}{ Automatable/Portable } \\
\hline $\begin{array}{l}\text { Lateral flow, sandwich } \\
\text { scheme, conductometric }\end{array}$ & Biosecurity & 79-1000 CFU mL & $10 \mathrm{~min}$. & \multicolumn{2}{|l|}{ Possible } \\
\hline SPR, optical, gold sensor & Medical & Down to $10^{6}$ cells $\mathrm{mL}^{-1}$ & $500 \mathrm{~min}$. & \multicolumn{2}{|l|}{ Neither } \\
\hline Incubated swabs, optical & Environmental & $\begin{array}{l}1000 \mathrm{CFU} / \mathrm{swab} \text { in } 10-13 \mathrm{~h}, \\
1-10 \mathrm{CFU} / \mathrm{swab} \text { in } 22 \mathrm{~h}\end{array}$ & $26 \mathrm{~h}$ & \multicolumn{2}{|l|}{ Portable } \\
\hline \multicolumn{2}{|c|}{$\begin{array}{l}\text { Flow-through, immmunofiltration } \\
\text { membrane, amperometric }\end{array}$} & Many & $50-1000$ cells $\mathrm{mL}^{-1}$ & 17 min. & Both \\
\hline
\end{tabular}

in conductance to determine the amount of bacteria present [23]. The working range of the sensor is from 79-1000 CFU $\mathrm{mL}^{-1}$ in liquid buffer, which is comparable with the sensor presented in this stydy. The total time of analysis is $10 \mathrm{~min}$, however, no studies have been done on the feasibility of making the system portable or automated and what the costs may be.

Another sensor uses surface plasmon resonance (SPR) to optically detect Salmonella and Listeria in liquid media [24]. The sensitivity of the sensor is comparable with a standard ELISA. However, the total analysis time is approximately $500 \mathrm{~min}$, making it much longer than the $17 \mathrm{~min}$ that has been achieved with the sensor developed in this study. In addition, the assay requires large, expensive equipment and the use of a gold sensor, which drives costs up and also prevents the system from being miniaturized or automated.

Yet another means of pathogen detection uses swabs and sponges to detect Listeria in the environment [25]. Upon being heated, a turbid solution is formed, allowing optical measurements to determine the number of bacteria present. The sensor can detect bacteria down to a limit of $1000 \mathrm{CFU} / \mathrm{swab}$ in 10-13 $\mathrm{h}$ and 1-10 $\mathrm{CFU} / \mathrm{swab}$ in $22 \mathrm{~h}$ wit $\mathrm{h}$ a total analysis time of $26 \mathrm{~h}$. This is a significantly longer amount of time for analysis than the sensor discussed is this study. The sensor is said to be portable, although the need for a long, heated incubation period probably prevents it from performing analysis in the field. A table comparing these recent advances in biosensors for the detection of bacteria is presented in Table 1 .

\section{CONCLUSION}

A new, amperometric immunoassay system has been optimized for rapid detection of low concentrations of E. coli. The design consists of a new immunosensor, which utilizes disposable immunofiltration membranes that also function as immunoelectrodes. These immunofiltration membranes help to eliminate some of the limitations of conventional immunoassays while also simplifying the assay procedure. The disposable immunofiltration membranes were constructed from Toray carbon paper upon which $E$. coli antibodies were immobilized using Woodward's Reagent K immobilization. Since the membranes are comprised of conductive carbon, they also function as an immunoelectrode, resulting in an effective increase in the local concentration of agents in the immunoreaction near the immunoelectrode, leading to the high sensitivity of the assay.

Indirect detection of the bacteria was accomplished using a sandwich scheme with HRP-labeled immunoconjugate [26]. Sodium iodide and hydrogen peroxide form the substrate of the peroxidase enzyme to produce iodine by the enzymatic oxidation of the iodide ions. The amperometric signal produced by the electroreduction of the iodine at the immunoelectrode was directly proportional to the amount of analyte present in a sample solution. Other parameters affecting the immunoassay performance, such as flow duration and immunoagent concentrations, were investigated and optimized.

The optimized immunoassay analysis time of $17 \mathrm{~min}$ was found to be shorter than the closest comparable immunoassay system. A flow rate of $200 \mu \mathrm{L} \mathrm{min}^{-1}$ was used to ensure that only small quantities of a sample would be required. A working range for the detection of total $E$. coli was found to vary linearly over analyte concentrations of 50-1000 cells $\mathrm{mL}^{-1}$. This is more sensitive than conventional detection techniques and allows for complete monitoring of bacteria concentrations over most government-approved health limits. The system should also be readily adaptable to a wide range of analytes, including other pathogenic bacteria, biological warfare agents, contagious and deadly viruses, as well as other biochemical species, such as drugs, hormones, or allergens.

\section{ACKNOWLEDGEMENTS}

The authors gratefully acknowledge the financial support of this study by the National Science Foundation. Appreciation also goes to the University of New Mexico School of Engineering for additional financial support. The authors would also like to thank Sagar Yelleti, Sireesha Chemburu and Dr. Jasmin Shah for their assistance.

\section{REFERENCES}

1. Brenner, D.J., 1984.Manual of Systematic Bacteriology. Williams and Wilkins, pp: 408-516. 
2. Ewing, W.H., 1986. Edwards and Ewing's Identification of Enterobacteriaceae. Elsevier Science Publishing, $4^{\text {th }}$ Edn.

3. Farmer, J.J. and M.T. Kelly, 1991. Manual of Clinical Microbiology. ASM, $5^{\text {th }}$ Edn., pp: 360-383.

4. Schaberg, D.R., 1991. Major trends in the microbial etiology of nonoscomial infection. American J. Med., 91: 72S-75S.

5. Blattner, F.R., G. Plunkett and C.A. Block et al., 1997. The complete genome sequence of Escherichia coli K-12. Science, 277: 1453-1474.

6. Farmer, J.J., B.J. Howard and A.S. Weissfield, 1987. Enterobacteriaceae. Clin. Path. Microbiol., pp: 289-328.

7. Siitonen, A., 1992. Escherichia coli in fecal flora of healthy adults. J. Infect. Dis., 166: 1058-1066.

8. Adam, A., 1923. Biology of colon bacillus dyspepsia and its relation to pathogenesis and to intoxication. Jahrb Kinderberth, 101: 295.

9. Ericsson, D.D. and J.L. DuPont, 1985. Traveler's diarrhea, recent developments. Current Clinical Topics in Infectious Diseases, pp: 66-84.

10. Strockbine, N.A., L.R. Marques and R.K. Holmes et al.,1985.Characterization of monoclonal antibodies against shiga-like toxin from Escherichia coli. Infection and Immunol., 50: 695-700.

11. Riley, L.W., R.S. Remis and S.D. Helgerson, 1983. Hemorrhagic colitis associated with rare Escherichia coli serotype. New England J. Med., 308: 681-685.

12. Stamm, W.E. and M. Turck, 1983.Urinary tract infection. Adv. Internal Med., 28: 141.

13. Fowler, J.E. and T.A. Stamey, 1977. Studies of introital colonization in women with recurrent urinary tract infections. J. Urol., 117: 472.

14. Mandell, G.L., R.G. Douglas, J.E. Bennet and R. Dolin, 2000. Principles and Practice of Infectious Diseases. Churchill Livingstone, $5^{\text {th }}$ Edn., pp: 823-837.

15. Abraham, S.N., E.H. Beachey and W.A. Simpson, 1983. Adherence of Streptococcus pyogenes, Escherichia coli and Pseudomonas aeruginosa to fibronectin-coated and uncoated epithelial cells. Infections and Immunol., 41: 1261.
16. McCracken, G.H. and I.D. Sarff, 1974. Current status and therapy of neonatal Escherichia coli meningitis. New England J. Med., 290: 1216-1220.

17. CDC, 2003.Website: http://www.cdc.gov/ncidod/dbmd/diseaseinfo/esch erichiacoli_g.htm

18. Toray Data Sheet, 2003. Toray Composites (America), Inc., 19002 50 ${ }^{\text {th }}$ Ave. E., Tacoma, WA 98446.

19. Krishnan, R., A.L. Ghindilis, P. Atanasov and E. Wilkins, 1995. Fast amperometric immunoassay utilizing highly dispersed electrode material. Anal. Lett., 28: 2459-2474.

20. Abdel-Hamid, I., 1999. Development of a portable immunoassay system for rapid and sensitive analyte detection. Ph. D Thesis. University of New Mexico.

21. Abdel-Hamid, I., D. Ivnitski, P. Atanasov and E. Wilkins, 1999. Flow-through immunofiltration assay system for rapid detection of $E$. coli O157:H7. Biosensors and Bioelectronics, 14: 309316.

22. Chamburu, S., 2003. An electrochemical biosensor for detection of pathogenic bacteria. M. Sc. Thesis. University of New Mexico.

23. Muhammad-Tahir, Z. and E.C. Alocilja, 2003. A conductometric biosensor for biosecurity. Biosensors and Bioelectronics, 18: 813-819.

24. Koubova, V. and E. Brynda et al., 2001. Detection of foodborne pathogens using surface plasmon resonance biosensors. Sensors and Actuators, 74: 100-105.

25. Firstenburg-Eden, R. and L.A. Shelef, 2000. A new rapid automated method for the detection of Listeria from environmental swabs and sponges. Intl. J. Food Microbiol., 56: 231-237.

26. Carnes, E.C., 2003. Development of a new design of immunosensor for the rapid and sensitive detection of bacteria. M. Sc. Thesis. University of New Mexico. 